\title{
Teleduca, un referente en cine educativo como reivindicación colectiva del espacio social. La experiencia de Carme Mayugo
}

Ricard HUERTA. Universitat de València (España). ricard.huerta@uv.es

Durante una estancia de investigación en la Universitat de Barcelona, y con el asesoramiento impagable del profesor Fernando Hernández (2016), pude acercarme a una realidad que me resultó fascinante: los nuevos modelos de mediación educativa que están funcionando en esta ciudad de efervescencias culturales. Las personas a quienes llegué a entrevistar me mostraron un panorama riquísimo y diverso, algo que todavía tiene más mérito si valoramos la situación de crisis que tan duramente ha afectado a los sectores culturales catalanes en general debido a las represalias por el denominado "procés". Entre las profesionales entrevistadas está Carme Mayugo, una pionera del cine educativo, con quien pude compartir experiencias (Garcia-Raffi y Jardon, 2018). La elección de la entrevista se debe también a la coincidencia con las IX Jornadas Internacionales de Investigación en Educación Artística "Cinema i Art als Entorns Educatius", unas jornadas que se celebran en Valencia los días 8 y 9 de noviembre de 2018. La propia Carme Mayugo es ponente en este encentro académico, donde se debaten las actuales situaciones que vive el audiovisual en el ámbito escolar y en otros entornos no formales. Desde el grupo Creari (GIUV2013-103) fomentamos la innovación mediante estos encuentros académicos en los que reflexionamos sobre arte y educación (Huerta, Alonso-Sanz y Ramon, 2018). Considero que las aportaciones de Mayugo son muy consistentes, tanto por la experiencia acumulada durante décadas por esta gran profesional, como por su propio relato personal, un relato que refleja una realidad nada fácil para quienes nos dedicamos a la cultura, las artes y la educación (Huerta y Alonso-Sanz, 2017). La entrevista se grabó en vídeo, y tuvo lugar en l'Hospitalet de Llobregat el 22 de noviembre de 2017.

Ricard Huerta. ¿Qué es Teleduca?, ¿Cómo están implicados los campos del arte y la educación en los procesos que promueve, y qué tensiones internas y externas genera trabajar como vosotros lo hacéis? 
Carme Mayugo. En Teleduca siempre nos hemos definido como un colectivo profesional interdisciplinario. Ya de forma constitutiva estamos formados por gente que viene de tres o cuatro campos con interés en trabajar la intersección entre ellos. Uno es el campo educativo desde una perspectiva no directiva, aunque veníamos del aula, pero con la intención de no intervenir, acercándonos a la educación artística y la educación audiovisual de tradición comunitaria o alternativa. Estas tres ramas confluyeron al encontrar un espacio que aquí en España era poco conocido o poco reconocido, pero que en los años 1990 se rescató desde la educación en comunicación. Nosotros veníamos de la tradición latinoamericana (representada especialmente por Mario Kaplún), no tanto de la anglosajona.

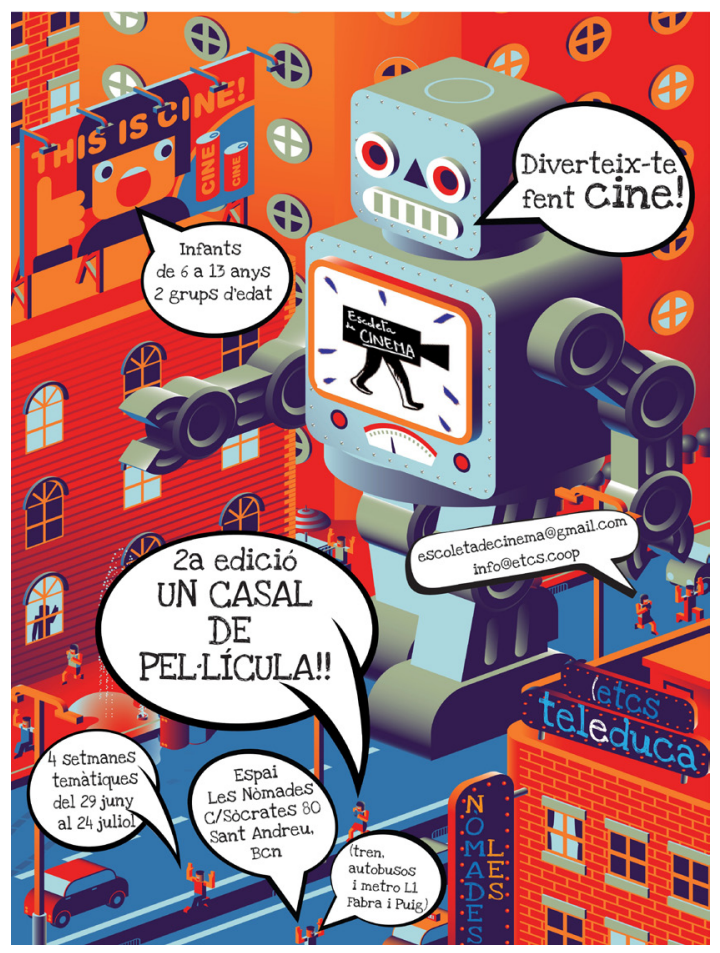

Figura 1. Cartel de la actividad "Un casal de pel·lícula"

En este espacio de comunicación siempre nos hemos interesado por ser un colectivo de intervención y de acción. En algún momento también nos hemos dedicado a la formación del profesorado (para que luego sea el profesorado quien haga), pero nos costaba mucho. Era como que tú pasabas ideas y maneras de hacer al profesorado, entonces el profesorado te pedía recetas, lo que a nosotros no nos interesaba, sino que preferíamos la intervención directa. Nos dimos cuenta que intervenir en el aula de secundaria (el audiovisual como herramienta de creación y de exploración de la identidad, de la relación con el entorno, de la conexión con la comunidad) en realidad nos llevaba fuera de la escuela. Entonces empezamos a trabajar en proyectos que conectaban los centros educativos con el barrio. Esto nos puso a trabajar con los barrios, con los servicios educativos (servicios juveniles, centros cívicos) que se estaban montando en los barrios a finales de los 1990 y principios de 
los 2000. Comenzamos entonces a trabajar las necesidades de cohesión social que había en estos barrios, con la gente que necesitaba generar servicios, íbamos donde se pudiera hablar de que aquello que realmente queríamos hacer.

En los inicios de los 2000 la gente nos llamaba, pero no para hacer creación audiovisual y educación, sino porque sabían que había una necesidad sobre la que se tenía que intervenir y no sabían cómo hacerlo. - "Teleduca hace unos vídeos y unas cosas que quedan muy bien, los hacen de manera participativa y la gente se pone en relación, y de ahí salen cosas..." Lo que más nos caracteriza son los procesos en el aula de manera divertida para aprender a hacer, y por otro lado dar un servicio en el territorio para que la gente hable y se conecte y se interpele de manera diferente, utilizando lo que sería la creatividad dialógica como centro de interés. Nosotros también descubrimos tarde que estábamos haciendo creatividad (cuando ya llevábamos cuatro o cinco años funcionando), porque estábamos en el marco de la educación y la comunicación. Estuvimos invitadas a un grupo de trabajo que generó la Fundación Jaume Bofill para reflexionar sobre el eje creaciónjóvenes-comunidad. Salió una publicación en la que participamos. Esto fue el origen de Artibarri (a principios de los años 2000). Fue al participar en este grupo de trabajo cuando nos dimos cuenta de que no sólo estábamos juntando educación y comunicación, sino que además hacíamos cultura, hacíamos creación artística, algo que inicialmente no nos habíamos propuesto.

Nosotras tenemos un posicionamiento que no es tanto de gestión cultural para hacer educación, sino que es al revés, somos educadoras que nos ponemos en el campo de la cultura. Es como una mirada, como una manera de ponerte en un lugar pensando hacia dónde miras y hacia dónde vas. Creo que cuando intervenimos comunicativamente lo hacemos incluso con una mirada muy pedagógica, en un sentido amplio.

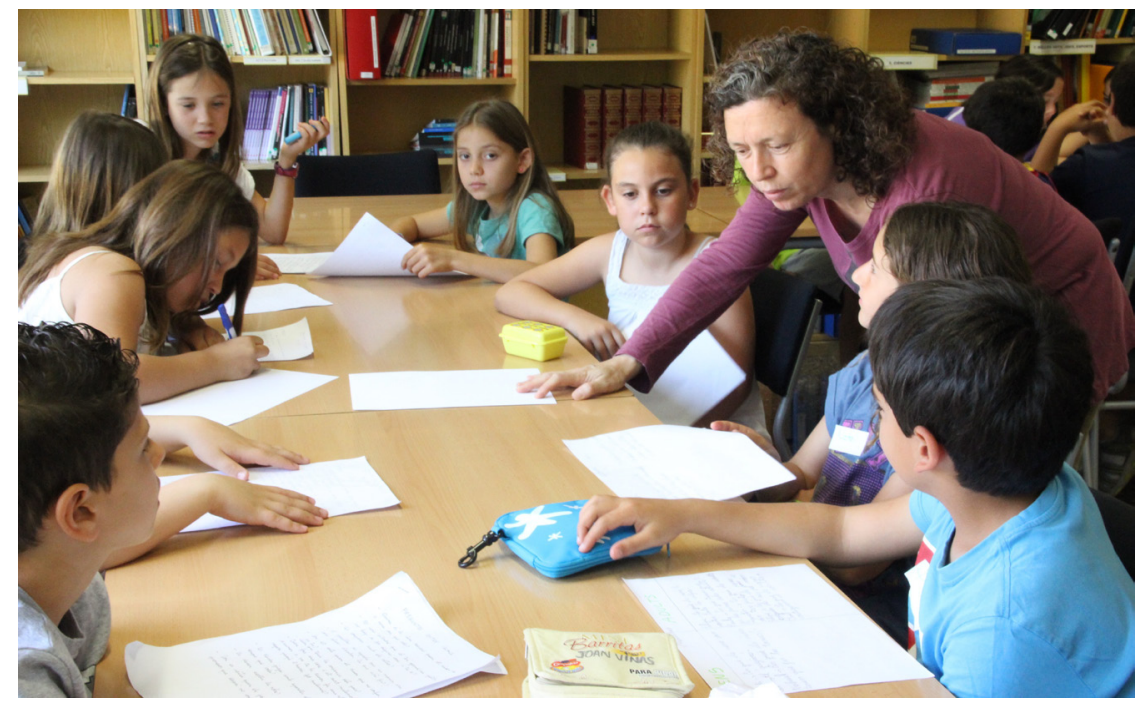

Figura 2. Carme Mayugo asesorando al alumnado de EDE Castelldefels para realizar el guión de un video. 
Ricard Huerta. ¿Cómo han evolucionado las estrategias en la trayectoria de Teleduca desde que nació la entidad?

Carme Mayugo. Nos hemos ido juntando con los compañeros de creación artística a partir de lo que comenzó en el proyecto Artibarri. Somos una de las entidades fundadoras. Se montó en 2002 (aunque oficialmente aparece en 2005). Siempre hemos pensado que es muy importante no ir solas. Cada cual tiene su camino para abordar este campo desde el espacio que conoce. Nosotras sabemos hacer audiovisuales, trabajar la comunicación, hemos trabajado en radio, con mujeres el tema de la fotografía, pero también queremos aprender de qué manera podemos ir generando democracia cultural, poco a poco, de pequeña acción en pequeña acción. No pretendemos democratizar la cultura o hacer llegar la cultura a la gente, sino que acompañamos a la gente a ser consciente de que puede generar cultura, y de que la cultura verdadera es la que genera la propia gente a través de poner en valor sus capacidades. Aunque nacimos trabajando mucho con adolescentes, nos hemos ido moviendo con los colectivos sociales, hemos trabajado bastante con mujeres (siempre hemos tenido una trayectoria de género, todas somos mujeres en el equipo). Nos pusimos a trabajar en temas sociales y más concretamente de emigración cuando prácticamente nadie casi trabajaba, a finales de los años 1990, en varias comarcas. Hubo un momento que nos planteamos trabajar con niños. Con los adolescentes ya nos encontrábamos muchas cosas que las teníamos vistas, y pensamos que teníamos que conseguir trabajar con los más jóvenes, como llegar antes a ellos. Trabajar con niños fue una inquietud. Empezamos por los últimos cursos de primaria, para ir bajando la edad de manera progresiva.

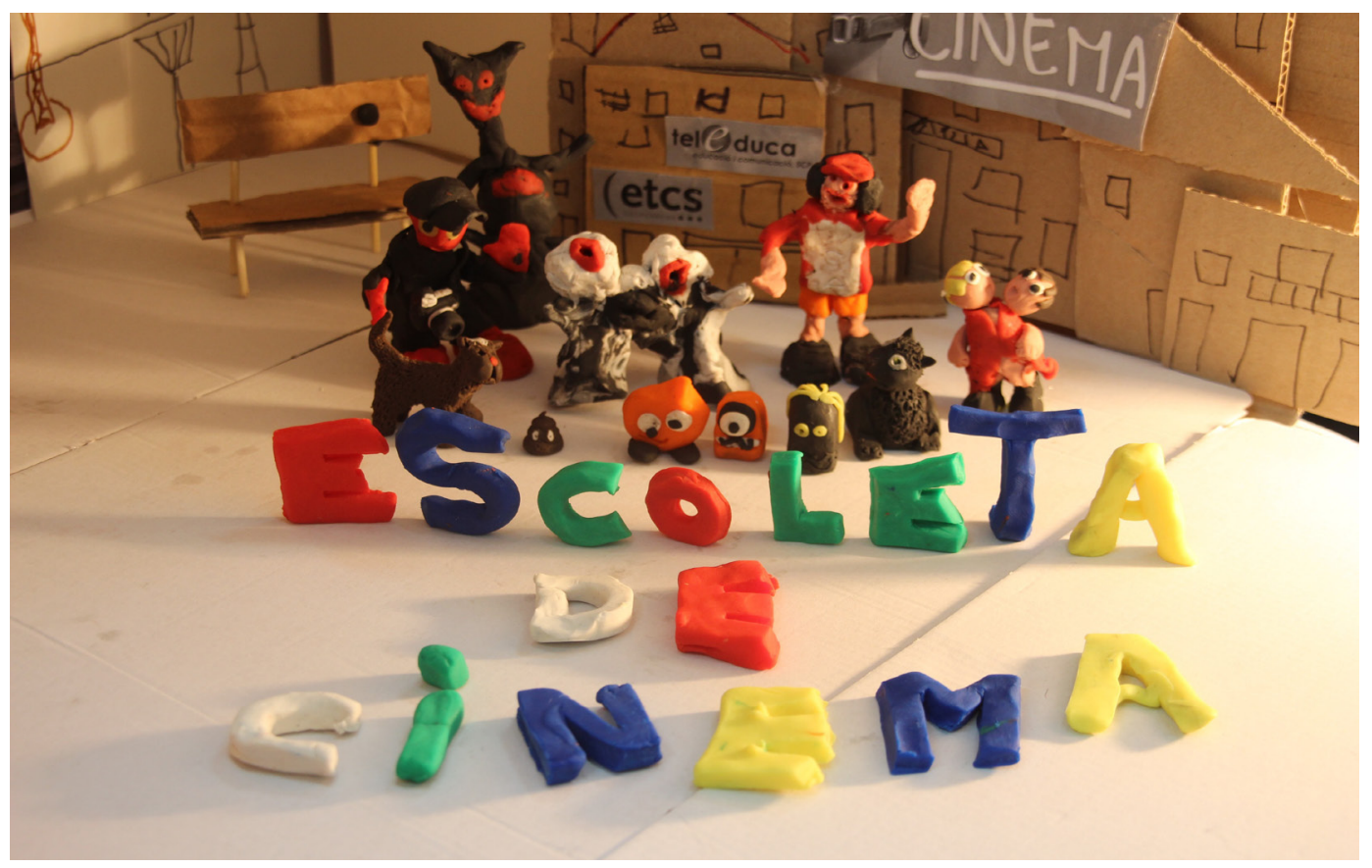

Figura 3. Figuras para elaborar un video mediante stop-motion como spot promocional de "Escoleta de Cinema". 
A veces hemos tenido retos a partir de las demandas que podían llegar. Lo que queremos es trabajar con toda la comunidad. Queremos interrelacionar grupos y colectivos. Esto ha sido una de las cosas que nos han ayudado a aprender. Teleduca no tiene proyectos que aplicar, sino que parte mucho de las demandas que llegan, $\mathrm{y}$ entonces idea un proyecto que sea a medida para dicha demanda. No generamos muchos proyectos propios (algunos sí que lo son) sino que vamos ajustándolos a cada caso. En el momento de la crisis nos ayudó mucho que nos llamaran otras entidades que querían este apoyo del vídeo. Querían a alguien que conociese bien cómo es la creación audiovisual con procesos participativos. Trabajamos mucho el traspaso entre la colaboración y la cooperación. Esto lo tenemos muy estudiado, en qué momento se puede ser más cooperativo, o en qué momento mejor ser más colaborativo, con qué grupos, hacer el traspaso del uno al otro.

Algo que viene del Mario Kaplún y que nos gusta mucho es el tema de partir de los conocimientos previos que tiene la gente para ponerlos en valor, no imponer qué tienen que decir o sobre qué tienen que hablar, sino que lo encuentren, como un hallazgo que la tienes dentro de ti. Cuando se hace un vídeo, la utilidad que cada persona le ve será la forma de partir para generar el vídeo, entendiendo cuáles son las necesidades (que la tienen que ver ellos).

Trabajamos mucho el tema del aprendizaje significativo, que lo que tú haces te implicas, tanto individualmente como a nivel de colectivo.

Ricard Huerta. ¿A nivel organizativo sois una empresa?

Carme Mayugo. Al principio éramos cuatro socias. Después una se fue, y es cuando incorporamos a Marta Ricart. Primero fuimos un grupo de trabajo, entre 1996 y 2000. En el año 2000 nos constituimos como Sociedad Civil Particular, que es lo que nos habían aconsejado desde el Colegio de Licenciados. Nadie confiaba en nosotros, teníamos que buscar un "nicho", nos preguntaban qué queríamos trabajar, si la educación o la comunicación, cosas bien raras. Hubo mucho tiempo (de 2000 hasta 2005) que era un trabajo de las cuatro socias para elegir un poco lo que serían las maneras de hacer, con cursos de formación, una serie de trabajos para hacerlos las cuatro. A partir de 2006 fue creciendo el volumen de trabajo y ya tuvimos como una parte laboral. Éramos cuatro socias con diferentes implicaciones, dos que trabajábamos de esto y dos que no, y empezamos a tener un equipo de personal contratado (llegamos a tener dos personas a tiempo completo y dos a media jornada). Comenzamos a experimentar un espacio que dirigíamos cuatro pero que compartíamos con los otros que eran trabajadores pero que queríamos que opinaran, que aprendiesen. Fue un aprendizaje constante, y fue madurando como proyecto, algo que duró hasta 2008, cuando llega el crash de la crisis. Como habían pasado tres años habíamos generado contratos fijos, y tuvimos que realizar despidos, que es lo más triste que te pasa en este tipo de proyectos. En esto no piensas nunca. Piensas 
que quizás no podrás crecer mucho, o que habrá momentos más bajos, pero no hasta el punto de desestructurar todo lo que habías montado.

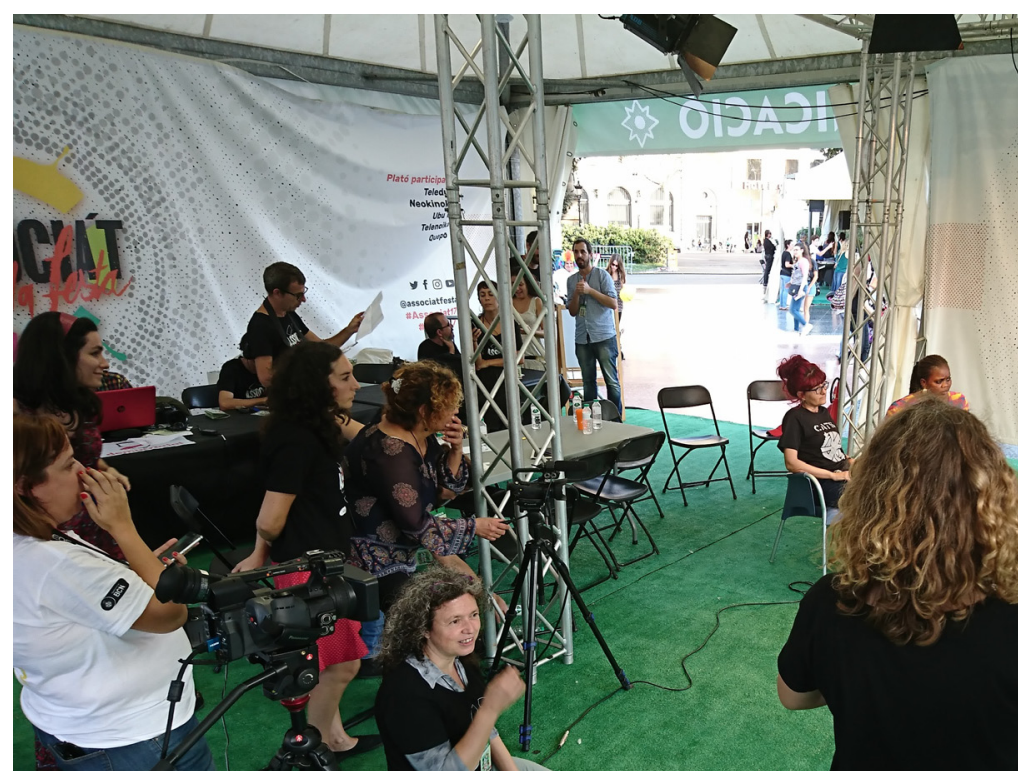

Figura 4. Grabando en el plató participativo de la actividad "Associa’t a la festa".

Ricard Huerta. ¿Cómo han sido las relaciones laborales entre vosotras?

Carme Mayugo. Entre 2009 y 2012 tuvimos que pasar a una etapa totalmente de resistencia. En 2010 marcharon tres de las socias, y me quedé yo sola, mientras que dos ex trabajadores se quedaron para ayudar. Empezamos a idear un sistema para trabajar todos como autónomos.

Un problema de Teleduca es el exceso de temporalidad. Tenemos unos meses de mucho trabajo y otros de muy poca, por lo que casi sólo da para una persona. En 2013-2014 se volvieron a afianzar los dos puestos y medio de trabajo que posteriormente hemos ido manteniendo. Los últimos años, como gestionamos un proyecto que ganamos por concurso (un ciclo de Cine y Derechos de los Niños), esto nos da más estabilidad. El último concurso con el Ayuntamiento de Barcelona tiene una duración de tres años. Era algo que nosotros no hacíamos (montar un ciclo de cine), y nos llegó por parte de la técnica del Ayuntamiento que llevaba este proyecto, una psicóloga que nos había visto trabajar a Sant Andreu y había coincidido con nosotros en más iniciativas.

Ricard Huerta. ¿Ha evolucionado la relación con las instituciones, teniendo en cuenta que se han vivido tiempos de crisis, y de post-crisis?

Carme Mayugo. Yo siempre explico que Teleduca no tiene ningún contacto político (esto es un desastre porque no nos reconocen el trabajo realizado), y creo 
que es porque hay mucho deseo de no tener ninguna vinculación con políticos. Y no hablo sólo por mí, ya que mis compañeras pensaban igual, cada una a su manera. Nos hemos definido como Colectivo Interdisciplinario Independiente (nunca mejor dicho). Nuestro gran bagaje han sido técnicos y técnicas de instituciones que fueran de donde fueran han llamado a Teleduca. Como ejemplo, hay una historia de una educadora juvenil que es con quien hicimos la primera experiencia con jóvenes, cuando aún estábamos montando Teleduca a finales de los años 1990. Ella siempre que ha tenido un proyecto nos llama. Incluso llegó a estudiar Comunicación Audiovisual. Actualmente es una dinamizadora juvenil que aplica la comunicación audiovisual. Ahora nos llama cuando necesita un apoyo.

Al principio funcionó muy por el boca oreja. Unos técnicos lo hablaban con otros técnicos. Esto cambió un poco a partir de 2008, cuando nacieron muchas otras asociaciones que hacían lo mismo que nosotros. Y está el tema de las tensiones. Nosotros ya conseguíamos que se nos pagase dignamente, mientras que los que llegaban buscaban trabajo, por lo que fuera, abaratando exageradamente los precios. Ahora por ejemplo, con una de estas asociaciones estamos colaborando, y yo siempre les recuerdo: cuando ustedes empezaron el 2008 nos tomasteis un proyecto para que nosotros éramos demasiado caras (así nos lo dijeron desde la entidad que promovía el proyecto). Nosotros presentamos un proyecto de $9000 €$, mientras gente que empieza llega a presentar presupuestos de $4500 €$ (se ríe). La precariedad...

Luego está la parte buena del asunto, y es que se da este reconocimiento de que llevamos veinte años con nuestra iniciativa y somos las que más tiempo hemos aguantado en ello, y por eso dicen: si hay alguien que sabe cómo se hace esto es Teleduca. Incluso intentamos llegar a acuerdos con las competidoras.

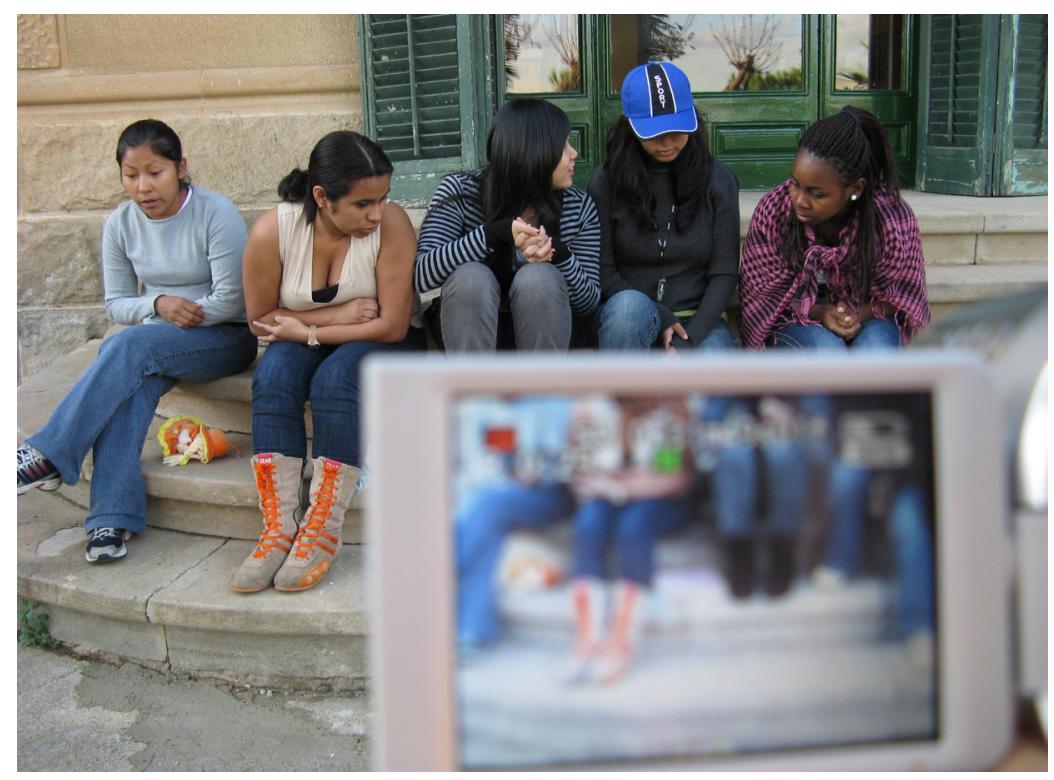

Figura 5. El trabajo con audiovisuales supone para los estudiantes de bachillerato un atractivo contacto con las tecnologías. 
Ricard Huerta. ¿Estáis ubicadas en Barcelona?

Carme Mayugo. Sí, pero también nos movemos en otros lugares. Nosotras hemos trabajado en una oficina técnica muy pequeña que tuvimos en el centro de la ciudad los primeros años, pero también en espacios ubicados en otros lugares. De 2000 a 2006 estuvimos en el centro. Estábamos en el centro para marchar hacia afuera, hacia las poblaciones de afuera, sobre todo provincia de Barcelona. Después apostamos por un local propio en el Camp del Arpa (El Clot, Barrio Iris), a un local que estaba abandonado. Lo rescatamos y estuvimos allí hasta 2014. En 2014 hubo un momento que podíamos despuntar, pero el local era como un lastre. No nos entendimos con la propietaria. En un momento que bajaban los alquileres no lo quería bajar. Y nosotros dijimos: si no nos pueden parar las instituciones, tampoco nos puede parar una propietaria (ríe de nuevo). Entonces tocó plegar velas, lo metimos todo en cajas, nos dividimos el despacho entre mi casa y casa del otro socio, el montador, es el socio con el que llevo más años juntos, ya que la tercera socia se fue a Argentina. Éramos los tres que habíamos quedado en el momento de resistencia (David, Paula y yo). Paula, en un momento que todavía no acabábamos de despegar, decidió volver a Argentina. Esto fue a finales del 2012, y cuando estaba a punto de irse a los inicios de 2013 es justamente cuando hacemos el despegue y empiezan a salir contratos. Ahora nos dice que nos echa mucho de menos. Paula fue la primera que verbalizó el problema, cuando me dijo: Carme, no estás obligada a tener un local, ya que no tenerlo te dará más libertad.

Ricard Huerta. ¿Qué experiencias destacarías como más significativas de los últimos años en Teleduca?

Carme Mayugo. La más significativa y en la que llevamos más años la iniciamos a comienzos de Teleduca, el año 2000, en Sant Andreu, y se ha ido transformando. Pero aún la estamos haciendo. Primero se llamó "Entra en la tele" y ahora se llama "Muestra de producciones audiovisuales de Sant Andreu". Es una experiencia en la promovemos la creación audiovisual en el aula en el ciclo superior de primaria. Participan 8 centros. Entran cada año los que hacen la actividad y nosotros hacemos el acompañamiento, con el profesorado para realizarlo, y luego un seguimiento. Después se hace también un encuentro entre los centros para compartir las producciones. Esta experiencia nos ha enseñado a trabajar con niños. Nosotras tenemos muchas experiencias con jóvenes. 


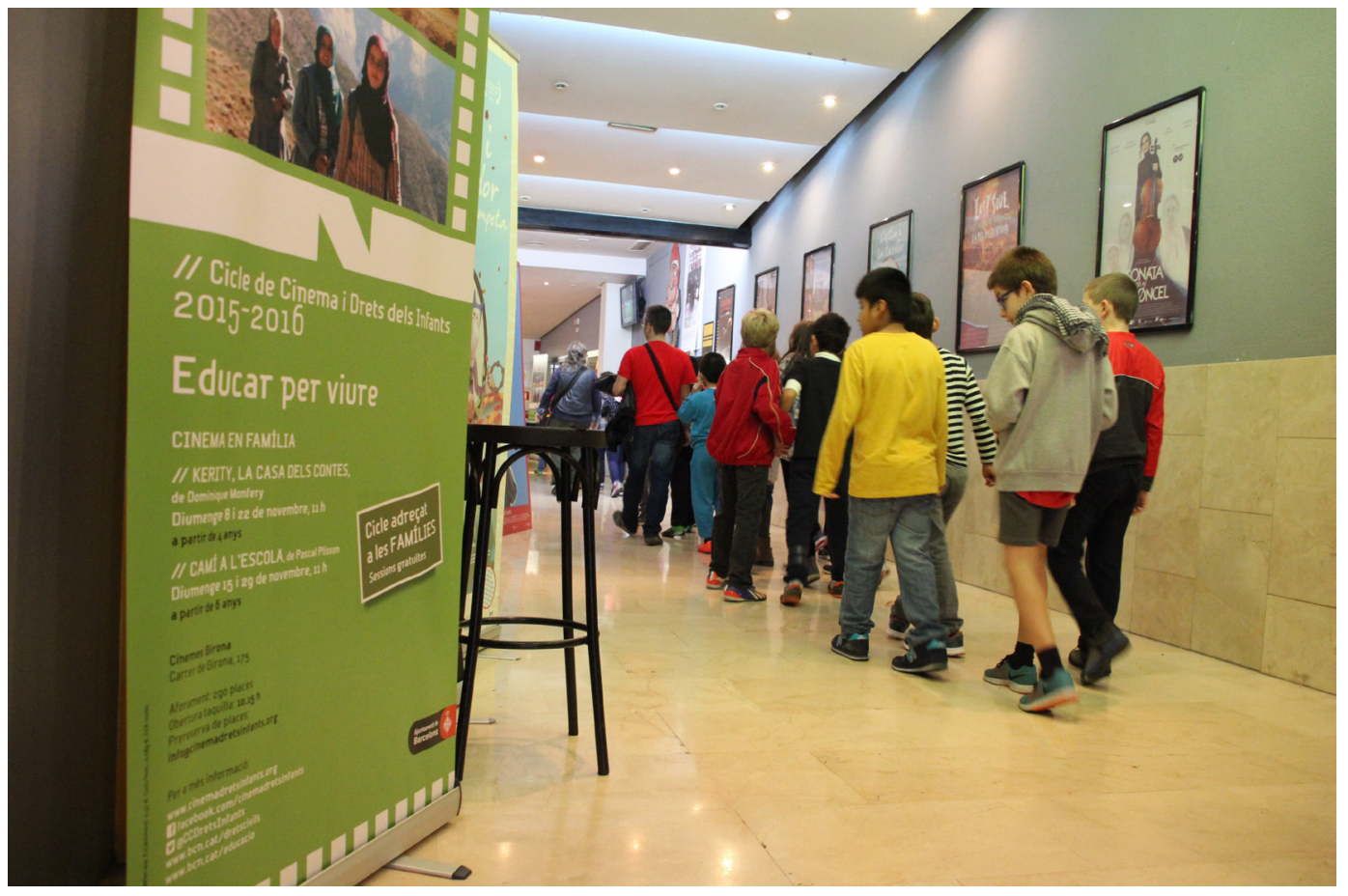

Figura 6. Alumnado asistiendo a las sesiones del "Cicle de Cinema i Drets dels Infants CCDI 2015 2016"

Otra experiencia que destacaría, porque es un lugar donde hemos vuelto a trabajar, es el Instituto Barri Besòs. A este instituto les propusimos hacer un proyecto europeo con un grupo de jóvenes como ellos, de Aix-en-Provence. Aquí trabajamos nosotros y una organización contraparte francesa que se llama Anonima. Resultó muy interesante vivir una experiencia para comprobar cómo entienden esto de la creación audiovisual participativa, por parte de una asociación que lo hace en Francia, y cómo lo trabajamos nosotros aquí. Hicimos el proyecto en paralelo. También acabamos fatal (ríe), puesto que los franceses lideraron el proyecto, pero nosotros la habíamos iniciado, y después se aprovecharon. Pero aprendimos. Lo que quedó de bueno es que los chicos y las chicas de este proyecto, que se llamó "Compartimos nuestras ciudades" (desde el punto de vista de las ciudades jóvenes), tenía como parte final la creación de un plató participativo dúplex. Al mismo tiempo, en Francia y aquí en un centro cultural, se realizaba un programa de televisión compartido, para ir pasando los vídeos que habíamos hecho unos y otros con el fin de relacionarlos. Nuestros jóvenes, que eran del barrio Besòs, no utilizan casi nunca el catalán, sólo cuando lo hablan en la hora de clase. La última semana decidieron que hablarían en catalán. Esto fue como un aprendizaje de cómo, cuando realmente pones las bases, las personas se pueden posicionar fuera de su marco, incluso si son adolescentes. Eran adolescentes de tercero de ESO, no es un momento fácil para hacer esto. Los profesores, cuando lo vieron, cuando vinieron a ver el programa, no se lo podían creer. 
Otra experiencia a destacar es "Les dones reporteres de Mataró" ("Las mujeres reporteras de Mataró"). En este caso fueron las mujeres quienes nos llamaron. Querían hacer un programa de radio, querían aprender a hacer radio. Las estuvimos acompañando entre 2008 y 2012. Ellas siguen haciendo la actividad, la actividad sigue viva, ya sin nosotros.

Una experiencia muy significativa es la realizada en un espacio rural. Fue en Mediona, en el Penedès, aplicando cosas que venían de otros proyectos, para hacer un proyecto intergeneracional con niños y personas mayores. Sirvió mucho para que, en un lugar donde había mucha dispersión territorial y mucha dispersión de grupos de población, comprobásemos de qué modo un proyecto de este tipo puede conseguir que la gente se encuentre. Fue muy potente. Actualmente siguen trabajando, y aunque nosotros ya no trabajamos allí, ellos se acuerdan de nosotros y nos van informando.

Y finalmente destacaría uno que hicimos hace muchos años en Sant Joan de Vilatorrada, junto a Manresa, que también tenía ese vínculo entre adolescentes, gente de un aula de acogida, y personas mayores de una escuela de adultos. Este binomio no lo hemos vuelto a repetir pero fue muy interesante, ya que trataba sobre las experiencias migratorias de unos y otros. Descubrimos el potencial de este tema. Había un vídeo al final que decía "A 15 horas de Sevilla y 10 horas de Pekín". Descubríamos que habían tardado más tiempo en llegar a Barcelona quienes venían de Sevilla que quienes venían de Pekín. Estas serían un poco las bases sobre las que hemos aprendido.

"Teleduca, educació i comunicació" es un colectivo independiente e interdisciplinar de profesionales provenientes de diversas especialidades relacionadas con la educación y la comunicación. Desde 1996 está llevando a cabo y consolidando un proyecto de Educación en Comunicación dirigido a diferentes públicos y colectivos. Uno de sus miembros más destacados es Carme Mayugo. Periodista de formación y con experiencia en TV, radio y prensa, ha sido durante seis años coordinadora de Clot-RTV Sant Martí, productora audiovisual independiente de documentales, además de organizadora de talleres de radio y televisión. La web de "Teleduca, educació i comunicació" es http://teleduca.org

\section{Referencias biblográficas}

Garcia-Raffi, V. y Jardon, P. (Coords.). (2018). Identidades, cine y educación. Valencia: Tirant lo Blanch.

Hernández-Hernández, F. (2016). Teaching the unknown to facilitate the emergence of a pedagogical event, Visual Inquiry: Learning \& Teaching Art, 5 (1), 87-95. doi:10.1386/vi.5.1.87_1 
Huerta, R. y Alonso-Sanz, A. (Coords.). (2017). Entornos informales para educar en artes. Valencia: Tirant lo Blanch.

Huerta, R. Alonso-Sanz, A. y Ramon, R. (Coords.). (2018). Investigar y educar en diseño. Valencia: Tirant lo Blanch. 